Interdisziplinäre Arbeitsgruppe Gentechnologiebericht:

Sina Bartfeld, Stephan Clemens, Tobias Erb, Heiner Fangerau, Boris Fehse, Jürgen Hampel, Ferdinand Hucho, Martin Korte, Stefan Mundlos, Jens Reich, Silke Schicktanz, Jochen Taupitz, Jörn Walter, Eva Winkler und Martin Zenke

\title{
Kernaussagen und Handlungsempfehlungen zu Organoiden
}

Die von der IAG Gentechnologiebericht verantworteten Kernaussagen sind folgendermaßen strukturiert: Zuerst erfolgt eine Zusammenfassung des aktuellen Stands und ein Ausblick auf die zukünftige Bedeutung von Organoiden für Forschung und Gesundheitsversorgung sowie ein Überblick über die Rechtslage und ethische Diskussion. Im zweiten Abschnitt werden hieraus Handlungsempfehlungen für die Politik abgeleitet.

\section{Kernaussagen zur Organoidtechnologie}

Aus Stammzellen abgeleitete Organoide

Organoide sind dreidimensionale, organähnliche Zellverbände, bei denen sich verschiedene Zelltypen so organisiert haben, wie es näherungsweise für das entsprechende Organ im Körper typisch ist. Sie weisen dabei drei Merkmale auf: Selbstorganisation, Vielzelligkeit und Funktionsfähigkeit. Die Bandbreite der Organe, die mit Organoiden erforscht werden können, wächst rapide an und umfasst u. a. Gehirn, Darm, Niere, Magen, Pankreas, Lunge, Leber, Prostata, Speiseröhre, Gallenblase und den weiblichen Reproduktionstrakt und daneben auch den Embryo (sogenannte Embryoide).

Organoide werden entweder aus pluripotenten Stammzellen oder aus gewebespezifischen adulten Stammzellen gezüchtet. Adulte Stammzellen sind in sehr vielen Geweben vorhanden und dafür zuständig, die Zellen in diesen Geweben zu erneuern. Sie können nur die Zelltypen hervorbringen, die in dem jeweiligen Gewebe vorkommen, z. B. produziert die Stammzelle des Darmepithels nur Zellen des Darmepithels (d. h. Deckgewebe und Drüsengewebe, das im Körper die inneren und äußeren Oberflächen auskleidet), aber keine Muskelzellen oder Nervenzellen. Sie sind damit multipotent. 
Im Gegensatz dazu können pluripotente Stammzellen alle der über 200 verschiedenen Zelltypen des menschlichen Organismus hervorbringen. Pluripotente Stammzellen werden entweder aus menschlichen Embryonen gewonnen (humane embryonale Stammzellen: hES-Zellen) oder durch eine sogenannte „Reprogrammierung“ von Körperzellen zu humanen induzierten pluripotenten Stammzellen (hiPS-Zellen) erhalten. Diese Körperzellen können von gesunden, aber auch von erkrankten Spenderinnen und Spendern stammen.

Beide Typen von Stammzellen, adulte und pluripotente, können in vitro dafür verwendet werden, Organoide zu züchten. Bei adulten Stammzellen wird dabei die natürliche Umgebung im Gewebe der jeweiligen Stammzelle nachgeahmt: Beispielsweise werden zu einer Stammzelle des Darmepithels die Signalstoffe hinzugegeben, die die Stammzelle auch in vivo im Darm umgeben würden. Als Reaktion auf diese Signale teilt sich die Stammzelle und bildet wie im Körper neues Darmepithel. Aufgrund der Ähnlichkeit zum echten Organ in vivo nennt man das in der Kulturschale entstehende Gebilde „Organoid“. Bei pluripotenten Stammzellen folgt man einem ähnlichen Prinzip, auch hier wird die natürliche Umgebung der Stammzelle in der Kulturschale nachgeahmt. Da aber diese Stammzellen ein viel breiteres Spektrum der Differenzierungsmöglichkeit haben, muss dabei in vitro eine Reihe von Entwicklungsschritten imitiert werden. Dafür wird in der Forschung das Wissen über die Embryonalentwicklung genutzt: In sequenziellen Schritten werden definierte Signalstoffe hinzugegeben, die auch im Körper dafür sorgen, dass die pluripotenten Stammzellen eine bestimmte Entwicklungsrichtung einschlagen. So wie aus Stammzellen in einem frühen Embryo erst nach vielen Entwicklungsschritten eine Darmwand wird, wird auch in der Zellkultur erst nach einigen Wochen aus pluripotenten Stammzellen eine Darmstammzelle, die dann ein Darmorganoid hervorbringt.

Die Verwendung von adulten vs. pluripotenten Stammzellen unterscheidet sich nicht nur in der Kultivierung der Organoide, sondern auch in ihren jeweiligen Vorund Nachteilen. Die Organoide aus adulten Stammzellen können bisher nur aus Epithel generiert werden und bleiben auch nur Epithel; so wird beispielsweise eine adulte Stammzelle des Darmepithels immer Darmepithel und nie Bindegewebe produzieren. Bei den aus pluripotenten Stammzellen abgeleiteten Organoiden ist das anders: Sie können auch andere Zelltypen enthalten, sind komplexer und heterogener - z. B. enthalten die so gewonnenen Darmorganoide durchaus Bindegewebe. Pluripotente Stammzellen können dadurch zu so unterschiedlichen Organoiden wie Darmorganoiden, Hirnorganoiden und Embryoiden differenziert werden. Hirnorganoide und Embryoide können dagegen durch die Technologie der adulten Stammzellen bisher nicht erzeugt werden. Daher resultieren für beide Technologien auch jeweils andere ethi- 
sche und rechtliche Überlegungen. Trotz dieser Unterschiede haben sie aber teilweise ähnliche Anwendungsgebiete.

Die Bedeutung der Organoidtechnologie für die Biomedizin

\section{Grundlagenforschung und Entwicklungsbiologie}

Der Einsatz von Organoiden ist für verschiedene Bereiche der Lebenswissenschaften vielversprechend. In der Grundlagenforschung können etwa die Steuerung der Organentstehung im Körper, die Zelldifferenzierung und die Stabilität von Geweben und Organen (Homöostase) untersucht werden. Dies gilt gerade auch für Gewebe, die in vivo bislang nicht oder nur schwer zugänglich waren, wie z. B. Hirngewebe. Ein großer Vorteil von Organoiden gegenüber zweidimensionalen Zellkulturen liegt darin, dass sie viele (idealerweise alle) Zelltypen enthalten, aus denen das Organ in vivo besteht. So können komplexe Vorgänge wie die Interaktion zwischen Zellen besser erforscht werden. Dementsprechend bilden Organoide auch krankheitsspezifische Merkmale besser ab als zweidimensionale Zellkulturen und zum Teil auch besser als Tiermodelle. Beispielsweise gibt es Krankheitserreger, die spezialisiert auf den Menschen sind und Tiere nicht infizieren. Solche Infektionskrankheiten können schon heute besser in Organoiden untersucht werden als im Tiermodell.

\section{Krankheitsmodelle}

HiPS-Zellen sind als Krankheitsmodelle seit Längerem Hoffnungsträger für die Grundlagenforschung und die personalisierte Medizin. Der Ansatz: Wenn von einer Patientin oder einem Patienten eigene Zellen gewonnen und vermehrt werden können, dann kann auch die jeweils eigene Pathologie erforscht und können für diese Patientin bzw. diesen Patienten passende Medikamente gefunden werden. Durch Organoide wird dies jetzt bereits klinische Realität. Die Organoidtechnologie ergänzt durch zwei entscheidende Weiterentwicklungen die iPS-Zell-Technologie: Erstens können durch die Organoidtechnologie jetzt auch direkt adulte Stammzellen von jeder Patientin und jedem Patienten vermehrt werden. Zweitens können in der dreidimensionalen Kultur komplexe Gebilde so geformt werden, dass Krankheiten besser modelliert werden können. Dadurch ermöglichen Organoide auch die Erforschung von genetisch bedingten Krankheiten oder Infektionen, die für Menschen spezifisch sind oder bei denen sich die Pathologie in Tiermodellen von der menschlichen Pathologie unterscheidet, sodass bislang keine guten Modellsysteme existieren. Ein möglicher klinischer Anwendungsbereich ist beispielsweise die Begleitdiagnostik im Sinne der personalisierten 
Medizin: Die Wirksamkeit eines Medikaments könnte direkt an aus patientenspezifischen Stammzellen abgeleiteten Organoiden getestet werden. Grundsätzlich ist das mit adulten und iPS-Zellen möglich, aber aufgrund des direkten Zugangs zu adulten Stammzellen werden diese bereits in der klinischen Diagnostik eingesetzt. In den Niederlanden ist eine organoidbasierte patientenspezifische Therapie bereits integraler Bestandteil der Behandlung von Mukoviszidose, und die Kosten für die Organoiddiagnostik werden dabei von den Krankenkassen übernommen. Organoide gewinnen auch in der Krebsforschung an Bedeutung. An Tumororganoiden können beispielsweise breit angelegte Screenings auf neue Krebsmedikamente durchgeführt werden. Daneben könnte die Entwicklung von Tumororganoiden einer Patientin oder eines Patienten zukünftig auch die individuelle Resistenzbildung gegen bestimmte Krebsmedikamente erforschbar machen. Klinische Studien hierzu sind vielversprechend. Durch die Möglichkeit der Nutzung von Organoiden als individuelle Krankheitsmodelle leistet die Organoidtechnologie einen wertvollen Beitrag zu einer personalisierten Medizin.

\section{Genetic Engineering}

Organoide können auch mittels unterschiedlicher Methoden, wie z. B. dem GenomeEditing (z. B. CRISPR/Cas), gentechnisch modifiziert werden. Genome-Editing bezeichnet Verfahren, bei denen einzelne DNA-Abschnitte (DNA: Desoxyribonukleinsäure), aber auch größere Genbereiche, aus dem Genom gezielt herausgeschnitten oder durch andere DNA-Abschnitte ersetzt werden. Dabei können aus einzelnen gentechnisch veränderten Zellen klonale Organoide mit den gewünschten genetischen Änderungen erzeugt werden, um etwa im Organoid den Effekt einer spezifischen Mutation zu untersuchen oder auch eine Mutation einer bestimmten Patientin oder eines bestimmten Patienten zu reparieren. Stammzellen können in einem mehrstufigen Verfahren in Zellkultur zuerst mithilfe von Genome-Editing-Verfahren modifiziert, dann vermehrt und in einem nächsten Schritt in das gewünschte Organoid differenziert werden. Das editierte Genom der Stammzellen wird bei der Differenzierung in Organoide an die Tochterzellen weitergegeben.

\section{Regenerative Medizin und Transplantation von Organoiden}

Eine zukünftige klinische Anwendungsmöglichkeit ist die Transplantation von Organoiden oder von aus Organoiden abgeleiteten Zellen in der Zellersatz- und regenerativen Therapie. Dabei sind Transplantate sowohl aus eigenem (autologem) als auch aus 
fremdem (allogenem) Material denkbar. Die Organoidtechnologie würde hier dazu genutzt werden, den gewünschten Zelltyp in den für eine Transplantation notwendigen Mengen herzustellen. Durch den zusätzlichen Einsatz von gentechnischen Methoden könnten zukünftig auch krankheitsauslösende Mutationen korrigiert werden, um gesunde Organoide für eine Transplantation zu differenzieren. Erste Experimente im Tiermodell lieferten bereits vielversprechende Ergebnisse, weitere Studien zur Funktionalität von Organoiden in vivo sind jedoch nötig.

\section{Biobanken}

Im Hinblick auf hiPS-Zellen ist bereits das Konzept der „lebenden Biobank“ bekannt. Dabei werden hiPS-Zellen von Spenderinnen und Spendern nach Gruppenmerkmalen sortiert eingefroren. Diese Gruppen können spezielle Patientengruppen sein („nur Patientinnen/Patienten mit Krankheit x“, ,alle Patientinnen/Patienten mit Operation $\left.\mathrm{y}^{\prime \prime}\right)$ oder gesunde Spenderinnen und Spender. Die Zellen können jederzeit aufgetaut und erneut vermehrt oder auch in Organoide differenziert werden. Zusätzlich gibt es jetzt auch die Möglichkeit, adulte Stammzellen in Form von Organoiden einzufrieren. Solche Organoidbiobanken wurden bereits weltweit für viele Organe aufgebaut, beispielsweise gibt es Biobanken von Darmorganoiden, Leberorganoiden und Organoiden der Nieren- und Harnwege. Sie können jeweils Organoide von mehreren Hundert Patientinnen und Patienten umfassen. Je nach wissenschaftlicher bzw. medizinischer Fragestellung können entweder spezielle Gruppen von Organoiden aufgetaut werden oder ganze Biobanken. Diese Banken haben eine besondere Bedeutung für Medikamententests: Die gelagerten Organoide können verwendet werden, um nach neuen Medikamenten zu suchen (Screening) oder die Bedeutung eines Medikaments für eine Gruppe von Patientinnen und Patienten zu testen (patientenspezifische Wirksamkeit); sie können auch für die Toxikologie eingesetzt werden.

\section{Toxikologie}

Bevor ein Medikament am Menschen getestet werden kann, muss untersucht werden, ob das Medikament potenziell toxisch für die Patientin oder den Patienten sein könnte. Dafür werden Tiermodelle verwendet, eine Ergänzung oder sogar Alternative könnten allerdings Medikamententests an Organoiden aus Biobanken sein. Es wird als sinnvoll erachtet, parallel Reihen von Organoiden zu testen und dabei besonders die häufig betroffenen Organe zu betrachten (Leber, Niere, Darm etc.). Die Aussagekraft von Organoidtests im Vergleich zu Tierversuchen für die Wirkung eines Medikaments in 
Patientinnen und Patienten wird derzeit erforscht. Es besteht die Hoffnung, dass in $\mathrm{Zu}$ kunft Toxizität und Wirkung an Organoiden getestet und so ein Teil der Tierversuche ergänzt oder sogar ersetzt werden könnten. Für Medikamententests vielversprechend ist auch die sogenannte Organ-on-a-Chip-Technologie, bei der in Zukunft mehrere unterschiedliche Organoide auf einem Chip zu einer Art reduziertem „Mini-Körper“ miteinander verbunden werden könnten.

\section{Grenzen der derzeitigen Organoidforschung}

Obwohl Organoide besonders im Vergleich mit herkömmlichen Zellkulturen eine frappierende Ähnlichkeit mit Organen aufweisen, sind sie derzeit (noch) stark reduktionistische Modelle und deutlich weniger komplex als entsprechende Organe. So besteht ein Darm im Körper nicht nur aus der inneren Schicht der Schleimhaut (die aus Epithel besteht), sondern ist umgeben von Bindegewebe und Muskelschichten, darüber hinaus von einem Nervensystem sowie von Blutgefäßen durchzogen und von Mikroorganismen besiedelt. Während Bindegewebe auch in aus pluripotenten Stammzellen abgeleiteten Organoiden vorhanden ist, fehlen oft die anderen Komponenten von In-vivoOrganen sowie von ihrer Umgebung im Organismus, die für das Funktionieren des Organs relevant sind. Nachteilig dabei ist, dass viele Wechselwirkungen - zumindest derzeit - noch nicht nachgebildet werden können. Es wird daher bereits an noch komplexeren Organoiden geforscht, etwa an Darmorganoiden mit einem funktionierenden Darm-Nervensystem. Menschlichen In-vivo-Organen möglichst ähnliche Organoide herzustellen, ist dennoch nur eines von verschiedenen Forschungszielen, denn der Vorteil der derzeitigen reduktionistischen Modelle ist, dass gezielt bestimmte Aspekte eines Organs und seiner Funktionen oder spezifische Effekte auf bestimmte Zelltypen untersucht werden können. Organoide stellen damit einen großen Fortschritt für die Biomedizin und vor allem für die aktuelle Forschung dar.

Ethische Aspekte der Forschung an Organoiden: Hirnorganoide, Embryoide und Mensch-Tier-Chimären

Die Organoidforschung ist mit zahlreichen ethischen Fragen verbunden, die beispielsweise bereits im Kontext der Embryonenforschung, der Forschung mit hES-Zellen, der Erzeugung von Mensch-Tier-Chimären und der Forschung mit Spendermaterial und -daten auch im Rahmen von Biobanken intensiv diskutiert werden. Daneben hat sie aber insbesondere in Bezug auf Hirnorganoide und Embryoide auch neue, für 
den Bereich spezifische Fragestellungen aufgeworfen. Diese ethische Diskussion steht insgesamt auch international noch am Anfang.

Aktuell sind die existenten Hirnorganoide noch weit von einem komplexen, menschlichen Gehirn entfernt. Aber es wurde vielfach die Frage aufgeworfen, ob in Zukunft komplexere Hirnorganoide oder miteinander fusionierte Organoide verschiedener Hirnareale (sogenannte „Assembloide“) ein Bewusstsein entwickeln könnten, und wenn, wie ein solches messbar wäre und welche ethisch-rechtlichen Schutzansprüche dann daraus abzuleiten wären. Dies könnte sowohl Hirnorganoide aus menschlichen als auch aus tierischen Zellen betreffen, wenn man davon ausgeht, dass auch viele höher entwickelte Tiere phänomenale Formen von Bewusstsein entwickeln. Vor dem Hintergrund, dass die Gehirnentwicklung im Menschen zudem bereits in frühen Entwicklungsstadien als normatives Kriterium für den ethischen und rechtlichen Schutz von Embryonen in vitro und in vivo angesehen wird, stellt sich außerdem die Frage, ob Hirnorganoide bzw. welche Entwicklungsstadien von Hirnorganoiden einem entsprechenden Schutz unterliegen müssten. Des Weiteren ergeben sich besondere ethische Fragen im Zusammenhang mit humanen Hirnorganoiden, die in lebende Säugetiere (z. B. Ratten, Mäuse, ggf. auch größere Säugetiere) transplantiert werden, um in vivo deren Interaktion mit anderen Geweben zu fördern und zu untersuchen. Hierfür wird in der Fachliteratur auch der Begriff „Mensch-Tier-Chimäre“ verwendet. In diesem Fall stellt sich nicht nur die Frage nach der normativen Bedeutung von Speziesgrenzen, wie sie bereits in anderen Bereichen der Stammzellforschung besteht, sondern auch danach, ob entsprechende Chimären dadurch unter Umständen intelligenter und/oder leidensfähiger würden und welche normativen Konsequenzen aus einer solchen Situation wiederum zu ziehen wären. Hinzu kommt, dass die Erzeugung von Mensch-Tier-Chimären auch im Kontext kulturgeschichtlicher Diskussionen um die Mensch-Tier-Grenzziehung bzw. deren Verschmelzung zu sehen ist, insbesondere wenn es um öffentliche und mediale Aufarbeitung geht.

Die Entstehung menschlichen Lebens ist bisher noch eine „Blackbox“ für die Forschung. Wie wird aus einer einzelnen Zelle, der befruchteten Eizelle, etwas so Komplexes wie ein Embryo? Die Forschung an menschlichen Embryonen in vitro ist in vielen Ländern aus ethischen, religiösen oder kulturellen Gründen entweder verboten oder eingeschränkt. In den letzten Jahren wurden aus murinen und humanen pluripotenten Stammzellen komplexe, organisierte Strukturen gebildet, die sehr frühen Stadien von Embryonen ähnlich sind. Die Forschung an Maus-Stammzellen ist dabei erheblich weiter als die an humanen Stammzellen. Manche Forscherinnen und Forscher gehen davon aus, dass es in absehbarer Zeit möglich sein wird, Strukturen zu schaffen, die nicht mehr von einem Embryo unterscheidbar sind. Die neuen Entitäten werden $u$. a. 
als „synthetische Embryonen“, „Embryoide“ oder auch „Blastoide“ bezeichnet. Diese sollten nicht verwechselt werden mit den schon lange bekannten „Embryoid Bodies“, die als undifferenzierte Aggregate von pluripotenten Stammzellen weniger komplex und organisiert sind und eine Vorstufe von Embryoiden bilden können. Aufgrund der Ähnlichkeit von Embryoiden mit menschlichen Embryonen werden diese derzeit in Übereinstimmung mit den rechtlichen Regularien vieler Länder zu menschlichen Embryonen nicht länger als 14 Tage kultiviert. Es ist aber eine offene Frage, wie Embryoide ontologisch einzustufen sind (so wie menschliche Embryonen oder anders?), wie sie entsprechend bezeichnet werden sollten und welcher normative Status ihnen zugeschrieben werden muss.

Diese offenen ethischen Fragen müssen auf der Basis einer interdisziplinären und gesamtgesellschaftlichen Debatte diskutiert werden, um politisch wie rechtlich konsensfähige Lösungen zu finden.

\section{Rechtliche Einordnung der Forschung an Organoiden}

Obwohl es in Deutschland keine spezifischen Rechtsregeln zur Herstellung und Verwendung von Organoiden gibt, werden sie doch sowohl vom Verfassungsrecht als auch vom einfachen Recht erfasst. Bezüglich der Herkunft des Ausgangsmaterials können Organoide insbesondere unter das Stammzellgesetz (StZG) fallen, das den Import und die Verwendung humaner embryonaler Stammzellen regelt. Die Gewinnung von hES-Zellen ist in Deutschland durch das Embryonenschutzgesetz (ESchG) verboten. Die Forschung an im Ausland generierten und nach Deutschland importierten hES-Zell-Linien ist zwar seit 2002 nach dem Stammzellgesetz zulässig, aber nur in begründeten Ausnahmefällen und unter strengen Voraussetzungen und außerdem nur für Forschungszwecke. Inzwischen mehren sich die Stimmen, die eine grundlegende Revision des Stammzellgesetzes fordern. So wird die Stichtagsregelung ebenso kritisiert wie das aufwendige Genehmigungsverfahren. Beides ist insbesondere auch verfassungsrechtlich problematisch. Sollten konkrete Therapien mit hES-Zellen verfügbar werden, dürfte zudem die Begrenzung der Verwendung von hES-Zell-Linien auf die Forschung nicht mehr haltbar sein - es sei denn, man würde inländischen Patientinnen und Patienten die Therapien vorenthalten wollen.

Die rechtliche Einordnung insbesondere von Embryoiden hängt davon ab, ob sie als menschliche Lebewesen mit einer Entwicklungsfähigkeit ähnlich der von menschlichen Embryonen eingestuft werden. Dann könnte ihnen unter Umständen Menschenwürde und Lebensschutz zuzuweisen sein. Ob sie schon lege lata vom Embryonenschutzgesetz erfasst werden, ist völlig ungeklärt. Jedenfalls rechtspolitisch wäre zu 
überlegen, ob ein so starker rechtlicher Schutz wegen der im Vergleich zu natürlichen Embryonen andersartigen Art der Herstellung unter Vermeidung einer Befruchtung, wegen der Entstehung in einem völlig anderen Kontext als der Erzeugung von Nachkommen und ggf. auch wegen der Absicht, die Entwicklung der entsprechenden Entitäten in einem sehr frühen Stadium zu beenden, zu verneinen wäre. Rechtspolitisch offen ist auch die Frage, ob weit entwickelte zukünftige Hirnorganoide denselben Regelungen wie Embryonen unterliegen sollten.

Mensch-Tier-Chimärenbildungen sind grundsätzlich sehr wenig durchnormiert. § 7 ESchG verbietet sie lediglich für den Fall, dass dabei menschliche Embryonen einbezogen werden oder durch Befruchtung einer menschlichen Eizelle mit dem Samen eines Tieres bzw. durch Befruchtung einer tierischen Eizelle mit dem Samen eines Menschen ein differenzierungsfähiger menschlicher Embryo entsteht; auch ist es verboten, einen so entstandenen menschlichen Embryo auf eine Frau oder ein Tier zu übertragen oder einen menschlichen Embryo auf ein Tier zu übertragen. Weitere spezielle Regeln enthält das deutsche Recht nicht; das Tierschutzgesetz regelt Tierversuche nur allgemein. Gerade bezüglich der Herstellung von Mensch-Tier-Organoiden oder auch vollständiger Mischwesen (etwa durch Implantation menschlicher Organoide in Tiere oder umgekehrt durch Einfügung tierischer Organoide in menschliche Individuen) existiert eine Reihe von Anwendungsszenarien, die den Ruf nach weiteren Vorschriften haben laut werden lassen. Zentral dürfte insbesondere die Forderung sein, dass entsprechende Forschung von einer auf diese Fragen spezialisierten Ethikkommission bewertet werden muss. Es kann nicht erwartet werden, dass die zur Genehmigung von Tierversuchen zuständigen Behörden und die zu ihrer Unterstützung tätigen (Ethik-)Kommissionen über ausreichende Fachkenntnisse zur Beurteilung der speziellen Fragen von Mensch-Tier-Chimären verfügen.

Mit Blick auf die grundrechtlich geschützten Persönlichkeitsrechte und die besondere Sensibilität von Gesundheitsdaten und genetischen Daten stellen sich je nach Verwendungskontext von Organoiden, die aus Zellen und Geweben von Patientinnen und Patienten bzw. Probandinnen und Probanden abgeleitet wurden, unterschiedliche datenschutzrechtliche Herausforderungen. Zwar ist die Rechtsgrundlage für die Verarbeitung personenbezogener und sensibler Daten, Gesundheitsdaten und genetischer Daten im medizinischen Behandlungskontext, beispielsweise im Zusammenhang mit dem Einsatz von Organoiden zu diagnostischen oder therapeutischen Zwecken, eindeutig bestimmbar, da sie in der Regel im Rahmen des Behandlungsvertrags vorgenommen wird. Allerdings gibt es verschiedene Rechtsgrundlagen, die bei der Verwendung entsprechender patientenspezifischer Daten für Forschungszwecke maßgeblich sein können. Das gilt auch für den weiteren Umgang mit Forschungsergebnissen, 
z. B. auch im Zuge der Lagerung bzw. Speicherung in einer Organoidbiobank. Hierbei stehen sich die allgemeinen Persönlichkeitsrechte (hinsichtlich des darin enthaltenen Schutzbereichs für personenbezogene Daten der/des Betroffenen) sowie die europäischen Datenschutzgrundrechte, vergleichbar konzeptualisiert, und die Forschungsfreiheit, gegenüber. Die genannten Rechte werden verfassungs- und unionsrechtlich geschützt. Während der Datenschutz eine informierte Einwilligung und damit hohe Anforderungen an die Spezifizierung von Forschungsvorhaben stellt, verlangt die Forschungsfreiheit einen möglichst umfassenden und einfachen Zugang zu Daten und Materialien auch für nur allgemein gehaltene Forschungsvorhaben. Diese beiden geschützten Rechtspositionen müssen in ein angemessenes Verhältnis zueinander gebracht werden. Die Abwägung der beiden Positionen könnte durch eine Handreichung der jeweiligen Datenschutzaufsichtsbehörde vereinfacht werden und Forschenden hierdurch mehr Rechtssicherheit ermöglichen. Diese Handreichungen wären insbesondere angesichts der Vielzahl an möglichen Rechtsgrundlagen ein wichtiger Beitrag für die Rechtsanwendung und Rechtssicherheit. 


\section{Handlungsempfehlungen für den Umgang mit der Organoidtechnologie}

Empfehlungen für Forschungsförderungen:

- Die Organoidtechnologie ist noch relativ neu und es ist zu erwarten, dass der zunehmende Erkenntnisgewinn zu neuen Anwendungen in der Biotechnologie, Biomedizin und in der Klinik führen wird. Die Forschung an Organoiden als Krankheitsmodelle und biotechnologische Testsysteme (einschließlich Organs-ona-Chip) sollte daher gezielt gefördert werden.

- Etablierung von Forschungsnetzwerken: Bei jeder neuen Technologie gibt es eine gewisse Vielfalt an Ansätzen. Um deutschlandweit gemeinsame Standards zu etablieren und starke Synergien $\mathrm{zu}$ ermöglichen, sollten insbesondere Forschungsnetzwerke, die Organoide als Krankheitsmodelle und biologische Testsysteme etablieren, validieren und standardisieren, gezielt gefördert werden.

- Einsatz in der Arzneimittelforschung: Organoide und Organoidbiobanken sind vielversprechend insbesondere für die Arzneimittelforschung. Es ist zu erwarten, dass sich Organoide sehr gut als Modelle für präklinische Wirksamkeits- und Toxizitätstests von Medikamenten eignen werden. Dabei werden neue Medikamente vor einem Einsatz am Menschen an Organoiden derjenigen Organe getestet, die für die Verstoffwechselung von Medikamenten eine zentrale Bedeutung haben wie Darm, Leber und Niere. Eine solche Verwendung könnte letztlich auch den Einsatz eines Teils der Tierversuche für diese Tests verringern, wenn nicht sogar ersetzen. Weiterhin können Organoide aus Biobanken verwendet werden, um bestehende Wirkstoffsammlungen nach neuen Medikamenten zu screenen oder Untergruppen von Patientinnen und Patienten zu identifizieren, die selektiv auf eine Behandlung ansprechen. Dies ermöglicht einen spezifischeren Einsatz von Medikamenten. Auch bessere Modelle für Krankheiten sind eine Grundlage für pharmazeutische Forschung, beispielsweise um Wirkmechanismen von Medikamenten zu verstehen und $\mathrm{zu}$ optimieren. Pharmazeutisch orientierte Grundlagenforschung und Kooperationen zwischen Forschung und Pharmaindustrie sollten daher gezielt gefördert werden.

- Translation in die Klinik: Die Organoidtechnologie hat ein hohes Potenzial für die personalisierte Medizin. Nach den Erfolgen in den Niederlanden sollte eine organoidbasierte personalisierte Medizin auch allen Mukoviszidose-Patientinnen und -Patienten in Deutschland alsbald zugänglich gemacht werden. Translationale Forschung und klinische Studien mit anderen organoidbasierten personalisierten Therapien, beispielsweise in der Krebstherapie, sollten gezielt gefördert werden. 
Die Möglichkeiten der Transplantation von Organoiden oder von aus Organoiden abgeleiteten Geweben sollte in der Grundlagenforschung untersucht werden. Da die für die Kultivierung von Organoiden wichtige dreidimensionale Matrix bisher nicht nach den Maßstäben der für die Anwendung am Menschen relevanten guten Herstellungspraxis (GMP) produziert wird, sind Transplantationen von Organoidmaterial in den Menschen noch ausgeschlossen. Die Suche nach alternativen Matrizes, die nach GMP-Standard produziert werden können, sollte daher gezielt gefördert werden.

- Einsatz von Genome-Editing-Verfahren: Auch für die Organoidtechnologie sollten die Techniken des Genome-Editings konsequent und langfristig erforscht werden, da sich hier neue Möglichkeiten zur patientenspezifischen Therapie und Medikamentenentwicklung (personalisierte Medizin) bislang nicht therapierbarer Erkrankungen eröffnen. Gleichzeitig sollten Sicherheits- und Risikoaspekte möglicher Anwendungen des Genome-Editings gründlich untersucht werden, da nur so eine fachkompetente Beurteilung und Abwägung der Chancen und Risiken für die Translation in die Klinik erfolgen kann.

- Interdisziplinäre Forschung: Da es sich bei der Entwicklung und dem Einsatz von Organoiden um Innovationen handelt, die weitreichende soziale, ethische und rechtliche Fragen aufwerfen und in Zukunft aufwerfen werden, müssen gezielt Forschungsprojekte gefördert werden, die diese Fragen interdisziplinär untersuchen. Bisher gibt es in Deutschland kaum ELSA-Forschungsprojekte zu Organoiden. Dabei wäre eine intensive Beteiligung von Forschenden aus den Geistesund Sozialwissenschaften vor dem Hintergrund der Vielzahl an offenen Fragen hinsichtlich konzeptueller, ontologischer, ethischer, rechtlicher und gesellschaftlicher Aspekte und Implikationen der Forschung an und Anwendung von Organoiden bedeutsam.

Ethische und rechtliche Empfehlungen:

- Forschung mit hES-Zellen: Auch für die Organoidtechnologie ist die Forschung mit hES-Zellen in absehbarer Zeit nicht durch die Forschung an hiPS- oder adulten Stammzellen zu ersetzen. Die Möglichkeit des Zugriffs auf hES-Zell-Linien auf dem derzeitigen Stand der Forschung ist wesentlich für deutsche Stammzellforscherinnen und -forscher. Die aus dem StZG resultierenden Beschränkungen der Forschungsfreiheit bezogen auf die Forschung mit hES-Zellen sind zudem ethisch umstritten und verfassungsrechtlich nicht gerechtfertigt. Aus diesen Gründen empfehlen wir nachdrücklich eine Aufhebung des durch das StZG festgeleg- 
ten Stichtags oder zumindest die Einführung eines gleitenden Stichtags. Damit deutsche Patientinnen und Patienten darüber hinaus auch von in Deutschland entwickelten medizinischen Anwendung der Organoidtechnologie profitieren können, ist es zudem notwendig, dass die Einfuhr und die Verwendung von hES-Zellen nicht nur zu Forschungszwecken, sondern auch zu diagnostischen, präventiven und therapeutischen Zwecken zulässig ist.

- Forschung an fetalem Gewebe: Um aus adulten und aus pluripotenten Stammzellen abgeleitete Organoide als wissenschaftliche Modelle der In-vivo-Umgebung absichern und weiter verbessern zu können, bedarf es ausführlicher Vergleichsstudien sowohl mit adultem als auch mit fetalem Gewebe. Hierfür sind Forscherinnen und Forscher auf den Zugang zu fetalem Gewebe, das andernfalls verworfen werden würde, angewiesen. Es bestehen in Deutschland bisher keine klaren rechtlichen Regelungen zu der Verwendung fetalen Gewebes und fetaler Zellen zu Forschungszwecken. Eine Verwendung der Zellen und Gewebe toter Feten muss im Falle eines Schwangerschaftsabbruchs unabhängig von der Entscheidung zum Abbruch erfolgen. Ebenfalls muss eine informierte Einwilligung der Schwangeren zu dem geplanten Forschungsvorhaben eingeholt werden. Von den Forschenden wird zudem ein verantwortungsvoller Umgang mit fetalem Gewebe erwartet.

- Embryoide: Die menschliche Embryonalentwicklung ist ein Forschungsfeld von zentraler Bedeutung für die Biomedizin. Embryoide bieten eine Möglichkeit, embryonale Entwicklungsprozesse auf Basis von Stammzellen in vitro nachzubilden und so der Forschung zugänglich zu machen. Momentan sind noch viele zentrale ethische wie rechtliche Fragen zum Umgang mit Embryoiden ungeklärt. Es müssen klare rechtliche Rahmenbedingungen für die Embryoidforschung geschaffen werden und bestehende Gesetze zur Forschung an menschlichen Embryonen ihrerseits überprüft werden. Dringend revisionsbedürftig ist z. B. das strikte Verbot der Forschung mit menschlichen Embryonen. Sie sollte in bestimmtem Umfang auch in Deutschland erlaubt werden, und zwar - nach erfolgter Zustimmung der biologischen Eltern - zumindest mit Embryonen, die zwar für Fortpflanzungszwecke erzeugt wurden, aber endgültig nicht mehr dafür verwendet werden und deshalb andernfalls verworfen werden. Es sollte auch eine zukünftig unter Umständen möglich werdende Nutzung von Embryoiden im Reproduktionskontext mit bedacht und in Anbetracht des Wohls des dabei entstehenden Menschen explizit verboten werden.

- Hirnorganoide: Bei Hirnorganoiden stellt sich die Frage, ob sie in Zukunft in der Lage sein könnten, ein Bewusstsein zu entwickeln. Es ist bisher weder theoretisch geklärt, welche Eigenschaften konkret unter den Begriff des Bewusstseins zu zäh- 
len sind (z. B. Selbstwahrnehmung, Empfindungs-/Leidensfähigkeit, Denken), noch praktisch, wie deren Vorhandensein zu messen wäre. Man sollte sich daher gemeinsam von neurowissenschaftlich-entwicklungsbiologischer und neurophilosophischer Seite um eine Schärfung und Differenzierung der Begriffe und Konzepte möglicher mentaler oder kognitiver Eigenschaften für Hirnorganoide bemühen; diese Diskussion sollte dabei von einer möglichst realistischen, forschungsstandorientierten Einschätzung des zukünftig Möglichen ausgehen. Darauf aufbauend muss geklärt werden, ob und welche ethisch-rechtlichen Schutzansprüche für menschliche und tierische Hirnorganoide und ggf. auch Tiere, auf die diese übertragen werden, daraus abzuleiten wären. Wichtig erscheint zudem, dass vor dem Hintergrund einer internationalen Fokussierung der wissenschaftlichen Debatte um Organoide auf diesen Aspekt nicht andere forschungsethische Fragen aus dem Blick geraten wie die mögliche Reduktion von Tierversuchen, die Aufklärung und Einwilligung von Spenderinnen und Spendern bzw. Patientinnen und Patienten in die Forschung sowie der ontologische, moralische und rechtliche Status der für die Herstellung von Organoiden verwendeten Zellen. In diesem Zusammenhang ist auch bei Hirnorganoiden die Frage zu stellen, ob sie in ihrem normativen Status demjenigen von menschlichen Embryonen anzupassen sind, sofern dieser Status auf einer frühen Gehirnentwicklung als Schutzkriterium basiert.

- Aufklärung und Einwilligung in die Forschung: Aufgrund der dynamischen Entwicklung von Forschungsprojekten und des Einsatzes neuer Technologien in der Organoidforschung sowie des Erfordernisses der breiten Verfügbarkeit und Vernetzbarkeit von Forschungsdaten in Biobanken ist eine informierte Einwilligung (Informed Consent) nicht immer umsetzbar. Eine „breite“ oder „dynamische Einwilligung" (Broad bzw. Dynamic Consent) könnten eine Alternative darstellen. Jedoch sind hierbei ethische Standards einzuhalten und insbesondere das Grundrecht auf Schutz personenbezogener Daten sowie das Selbstbestimmungsrecht von Patientinnen und Patienten bzw. Spenderinnen und Spendern sind zu gewährleisten. Daher empfehlen sich darüber hinausgehende Maßnahmen zur Steigerung der Transparenz, Datensicherheit und Vertrauensbildung, z. B. die Nennung von Datenverarbeitungsmethoden, Schutzmaßnahmen zur Minderung ihrer Risiken (wie z. B. technisch-organisatorische Zugangsbeschränkungen zu den gesammelten Daten), die Einrichtung einer Webseite zur Information der Studienteilnehmenden über das konkrete Forschungsprojekt und die Einräumung einer Widerspruchsmöglichkeit. Gerade äußerst sensible Forschungsinhalte wie z. B. die Forschung an hES-Zellen, fetalen Zellen und Geweben, Embryonen, Embryoiden sowie Hirnorganoiden sollten Gegenstand einer Einwilligung sein. In diesem Sinne sollte ein mit 
der Forschungsfreiheit im Einklang stehendes Höchstmaß an Entscheidungsmöglichkeiten der Teilnehmenden über die Verwendung ihrer Daten und Biomaterialien angestrebt werden.

- Reduktion von Tierversuchen: Die Nutzung von Organoiden als Krankheitsmodelle und Testsysteme für toxikologische Screenings hat das Potenzial, Tierversuche in Grundlagenforschung und pharmazeutischer Industrie zu ergänzen. Vor dem Hintergrund des international anerkannten Prinzips der 3R (,replacement, refinement, reduction“) und dessen Umsetzung durch die EU-Richtlinie „[...] zum Schutz der für Versuche und andere wissenschaftliche Zwecke verwendeten Tiere“ (2010/63/EU) ist es wichtig, dass Alternativmethoden entwickelt, validiert und dann eingesetzt werden. Hierfür sind auch die rechtlichen Vorgaben für den sogenannten Verbraucherschutz bezüglich Verträglichkeit und Toxikologie zu überprüfen und anzupassen.

\section{Empfehlung zur Aufklärung und Einbeziehung der breiten Öffentlichkeit:}

- Wissenschaftskommunikation: Gerade im Bereich der Stammzellforschung, die große Potenziale in sich birgt und dadurch auch mit großen Hoffnungen verbunden ist, hat sich eine verständliche, vorausschauende und realistische Kommunikation von wissenschaftlichen Ergebnissen und therapeutischen Möglichkeiten in den letzten Jahrzehnten als wichtig erwiesen. Hierbei sollten die verschiedenen Kommunikatoren wie Wissenschaftseinrichtungen, einzelne Forschende und Unternehmen sowie Vereine und Verbände eine angemessene Wissenschaftskommunikation anstreben, die sich an eine breite Öffentlichkeit wendet. Zu diesem Zweck sind (z. B. im Sinne der Guidelines der International Society for Stem Cell Research (ISSCR) von 2016) hohe Anforderungen an die Wissenschaftskommunikation über Stammzellen und daraus abgeleitete Organoide zu stellen. Wissenschaftskommunikation sollte immer auch Grenzen und Probleme der Forschung und Entwicklung aufzeigen, um keine übersteigerten Hoffnungen auf baldige Therapien seitens der Öffentlichkeit aufkommen zu lassen. Forschende und Wissenschaftseinrichtungen sollten zudem über einen ethischen Kodex einer angemessenen Kommunikation ihrer Ergebnisse nachdenken.

- Mögliche ungeprüfte Therapien mit Organoiden oder aus Organoiden gewonnenen Zellen: Weltweit besteht ein großes Angebot an ungeprüften Behandlungen mit Stammzellen, die nicht im Rahmen klinischer Studien auf ihre Sicherheit und Wirksamkeit geprüft wurden und die Gesundheit von Patientinnen und Patienten sowie den Ruf der Stammzellforschung gefährden. Es muss vermieden werden, 
dass es auch bei Organoiden zu verfrühten und wissenschaftlich nicht gerechtfertigten Anwendungen und Angeboten ohne behördliche Zulassung kommt. Die jeweils geltenden Bedingungen der Marktzulassung von Medikamenten und Therapien sollten überprüft werden, um geeignete Kriterien und Grenzen derselben zu etablieren. Dabei empfehlen sich auch die Entwicklung und Etablierung internationaler Standards für die Regulierung der klinischen Anwendung.

- Breite gesellschaftliche Debatte anstoßen: Die ethische und rechtliche Diskussion um Organoide steht gerade erst in den Anfängen. Es ist daher wichtig, frühzeitig eine breite gesellschaftliche Diskussion zu initiieren, die verschiedene Interessengruppen und die allgemeine Bevölkerung gleichermaßen einbindet. Hierzu können sowohl nationale Institutionen beitragen als auch die Förderung von Forschungsprojekten, die sich mit den ethischen, rechtlichen, sozialen und ökonomischen Bedingungen der Organoidforschung im Detail auseinandersetzen. Es ist dabei darauf zu achten, dass in der öffentlichen und medialen Debatte keine überzogenen Heilsversprechen gemacht werden, sondern eine sachliche, faktenbasierte Diskussion um die Möglichkeiten und Grenzen der Organoidforschung stattfindet. 